\title{
Aperçu des contributions des néogramsciens et des théories critiques au tournant réflexif des théories de la sécurité
}

Frédérick Guillaume Dufour

\section{(2) OpenEdition \\ Journals}

Édition électronique

URL : http://journals.openedition.org/conflits/1531

DOI : 10.4000/conflits.1531

ISSN : 1777-5345

Éditeur :

CCLS - Centre d'études sur les conflits lilberté et sécurité, L'Harmattan

Édition imprimée

Date de publication : 1 juin 2004

Pagination : 53-79

ISBN : 2-7475-7301-X

ISSN : 1157-996X

Référence électronique

Frédérick Guillaume Dufour, « Aperçu des contributions des néogramsciens et des théories critiques au tournant réflexif des théories de la sécurité », Cultures \& Conflits [En ligne], 54 | été 2004, mis en ligne le 08 janvier 2010, consulté le 30 mars 2021. URL : http://journals.openedition.org/conflits/1531 ; DOI : https://doi.org/10.4000/conflits.1531

Ce document a été généré automatiquement le 30 mars 2021

Creative Commons License 


\title{
Aperçu des contributions des néogramsciens et des théories critiques au tournant réflexif des théories de la sécurité
}

\author{
Frédérick Guillaume Dufour
}

Le champ des théories de la politique globale ${ }^{1}$ 'est transformé entre 1981 et 2001. Parmi ces changements, l'émergence et la diffusion des théories critiques ont joué un rôle central. Celles-ci se construisent sur des bases qui se veulent en rupture avec la manière de faire habituelle au sein de ce champ, étroitement lié, durant les années 1960-70, aux impératifs de la Guerre Froide. Parmi les théories critiques, on retrouve de nombreuses mouvances théoriques distinctes, voire antagonistes. Des variantes du constructivisme aux approches déconstructionnistes, en passant par une panoplie d'approches néomarxistes et féministes, le champ de la théorie de la politique globale n'a plus les mêmes contours qu'il y a vingt ans. Comme l'ordre mondial au sein duquel il évolue, ce champ s'est transformé en faisant émerger de nouveaux pôles de pouvoirs et de résistances.

L'expression théories critiques désigne l'ensemble de théories qui ont participé au mouvement de repositionnement du champ de la théorie de la politique globale durant les vingt dernières années. Dans une très large mesure, l'objet de cet article ce sont les contributions que les néogramsciens ont apportées à la transformation du champ des Relations Internationales. Plusieurs théories critiques ont intégrées ces contributions, mais l'ensemble des théories critiques est loin de se réduire aux contributions des néogramsciens. L'analyse néogram-scienne qui a commencé à se développer au début des années 1980 est devenue une des principales théories critiques des Relations Internationales. Bien que son noyau ait été situé à l'Université York à Toronto, à celle de Sussex en Angleterre, et à celle d'Amsterdam jusqu'au début des années 1990, elle s'est internationalisée avec le développement de nouveaux axes de recherche au Japon et en Allemagne. 
Dans ce qui suit, nous nous livrerons à la présentation du contexte matériel de la production des relations internationales durant les dernières années, des innovations ontologiques, épistémologiques et normatives des théories critiques, et les néogramsciens. Finalement, nous situerons ce qui nous semble être le contexte de production actuel auquel est confrontée la théorie néogramscienne.

\section{Conditions matérielles de l'énonciation de la théorie de la politique globale}

Comme l'a souligné Didier Bigo pour le monde de la sécurité, sans un contexte d'interprétation, on ne peut saisir les intentions des énonciateurs et la signification de leur discours ${ }^{2}$. Une formation discursive dominante au sein d'un champ ne peut pas être réduite au reflet idéologique des rapports de force constitutifs de la politique globale ; la dynamique interne du champ produit également des rapports de force qui co-influence la coloration des formations discursives. Parmi les discours savants, les discours sur l'économie politique des Relations Internationales et les études de la sécurité, sont des sous-champs où tout acte de langage peut avoir des conséquences importantes sur le champ politique. Ce sont des champs où dire, c'est faire. Bien souvent le discours sur la sécurité est plus politique que théorique, c'est-à-dire qu'il " vise à produire et à imposer des représentations du monde social qui soient capables d'agir sur ce monde en agissant sur la représentation que s'en font les agents $»^{3}$.

Notre propos porte sur la période où commencent à apparaitre de nouvelles formes de dissensions au sein du champ de la théorie de la politique globale, période dont nous situons l'émergence avec la publication, en 1981, de « Social Forces, States and World Order : Beyond International Relations Theory» de Robert W. $\operatorname{Cox}^{4}$. La théorie de la politique globale est dominée au début des années 1980 par une quête de scientificité qui ne sait plus à quelles sciences emprunter ses modèles explicatifs : fonctionnalisme, béhaviorisme, cybernétique, systémisme, et économie néoclassique se succèdent sans faire l'unanimité. Dans le contexte de sa production, l'article de Cox répondait à la reformulation de la théorie réaliste entreprise par Kenneth Waltz. Il tentait de souligner le caractère politico-normatif de certaines théories, dont le néoréalisme qui, malgré leur apparence de scientificité, n'en sont pas moins porteuses de conséquences normatives ou d'un projet politique. La période de la publication de cet article correspond, sur le plan politique à l'essoufflement, puis à l'inattendue renaissance, de l'hégémonie américaine, à l'accès au pouvoir de forces sociales néoconservatrices aux Etats-Unis, en Angleterre et en RFA, et à une transition accélérée d'une organisation fordiste à une organisation post-fordiste de la production.

Le savoir sur la politique globale ne peut pas être réduit à un discours qui serait imposé par une classe dirigeante. Comme tout autre champ, celui de la théorie de la politique globale est structuré par des procédures discursives plus subtiles, qui occupent une position centrale à une certaine époque, alors que d'autres procédures sont raréfiées ou délégitimées. Le fait que les classes dominantes diffusent certains slogans contribue à la diffusion d'une certaine représentation du monde, mais les professionnels de la politique globale énoncent leurs positions à l'intersection de beaucoup d'autres logiques que la stricte logique de la reproduction des classes. Les effets de mode, les liaisons avec le monde de la défense, les contraintes liées au monde des subventions, les possibilités d'avancement personnel académique et politique entrent en ligne de 
compte dans l'énonciation de la politique globale. Ce qui caractérise ce champ durant les années 1980, c'est le fait que l'institutionnalisme néolibéral et le néoréalisme s'installent dans un dialogue, le "débat néo-néo ", dont ils excluent leurs rivaux, en en raréfiant la participation. Ces deux théories partagent la même orientation épistémologique, elles s'accordent sur le choix de l'Etat comme unité d'analyse fondamentale, mais divergent sur la question de la coopération. Ceux qui travaillent à l'extérieur de la tradition de science normale sont délégitimés et qualifiés, selon des stratégies d'exclusion courantes, soit de radicaux, de réflectivistes ou d'interprètes de textes.

Les contradictions issues de l'expansion du mode de production capitaliste sont négligées par les néoréalistes et les institutionnalistes néolibéraux. Depuis la fin de la Guerre Froide, un des effets néfastes qu'eut le discours de la « fin de l'histoire » sur la pratique théorique est le fait qu'en raison de son aspect « inéluctable » le capitalisme ait cessé de faire l'objet d'une analyse systématique ${ }^{5}$. Contrairement à ce que plusieurs critiques de Fukuyama ont affirmé, nous estimons que celui-ci met le doigt sur quelques éléments importants du monde politique contemporain. Il fait davantage une apologie de l'Etat de droit, que du capitalisme néolibéral. Fukuyama n'a jamais été naïf au point de croire que l'effondrement du bloc de l'Est signifiait la fin des conflits sociaux dans un monde ayant adopté le modèle libéral. Il soutient même explicitement le contraire. Il estime que le modèle de l'Etat de droit libéral issu de la Révolution française sera probablement la dernière forme d'organisation politique à exister et à régler les conflits contemporains. L'Etat de droit, bien qu'il soit assez systématiquement bafoué dans la pratique, demeure un modèle théorique d'organisation politique (national ou éventuellement supranational) auquel on voit peu d'alternatives légitimes, crédibles et viables. Là où l'analyse inspirée de Fukuyama rencontre un problème, c'est quand elle s'appuie sur ce premier constat pour éviter de faire l'analyse du capitalisme avancé, du néolibéralisme ou du néocolonialisme. Non seulement ces idéologies demeurent des éléments clés de la compréhension du monde contemporain ${ }^{6}$, mais ce sont des éléments dont on aurait tort de croire qu'ils sont nécessairement concomitants du renforcement de l'Etat de droit, et c'est sans doute sur ce point que l'analyse de Fukuyama est la plus limitée. Un problème se pose alors, autant pour certains défenseurs, que pour certains critiques de Fukuyama. Ainsi, s'il est juste qu'en théorie, l'Etat de droit semble une organisation politique à laquelle on voit peu d'alternatives légitimes ${ }^{7}$, par contre, il reste à démontrer que l'expansion du mode de production capitaliste s'accompagne réellement d'une expansion de droits politiques et sociaux substantiels et non d'une simple globalisation de la liberté d'investissement, des trafics douteux, des abris fiscaux et des moyens de coercitions. Trop souvent l'économie néoclassique se soucie peu ou trop tard des effets politiques et sociaux de l'application de ses préceptes.

Dans la périphérie et la semi-périphérie, non seulement le néolibéralisme économique et le libéralisme politique (au sens où l'entend Rawls) ne convergent pas, mais ils tendent plutôt à se nuire. L'implantation du premier, quand elle ne se fait pas par la force, favorise l'essor de conditions sociales criminogènes. Bien sûr, les économistes néoclassiques ne nient plus que l'application de leurs politiques ait mené à certains ratés, qu'elle se soit parfois accompagnée de contrecoups écologiques et sociaux. Simplement, soutiennent-ils qu' « avec le temps » ces effets pervers se régleront d'euxmêmes. 
Le second spectre qui hante les approches «néo-néo» est la proximité entre les communautés de chercheurs et le pouvoir politique. Comme tout autre champ scientifique, celui-ci n'échappe pas au système des subventions des secteurs privés et publics, mais en Relations Internationales, l'impulsion des conseillers du Prince à vouloir voir les résultats de leurs recherches récupérés en haut lieu a tracé une ligne de démarcation très arbitraire entre les policy relevant research agendas (programmes de recherches utiles pour la formulation des politiques publiques) et le reste de la recherche. L'imbrication du champ académique états-unien dans le champ politique n'est pas étrangère à la délimitation des débats entre les institutionnalistes néolibéraux et les néoréalistes. Les deux produisent des policy relevant discourses auxquels Démocrates et Républicains sont loin d'être insensibles.

En théorie, l'autonomie du champ académique assure que le travail théorique soit contre examiné par une communauté de chercheurs, mais aucune recherche n'est entièrement détachée d'intérêts matériels et la privatisation du champ académique n'est pas sans menace pour l'intégrité académique ${ }^{8}$. C'est particulièrement vrai aux Etats-Unis où le budget consacré à la recherche dans le domaine de la sécurité dépasse de loin celui de tout autre Etat. Le champ de la politique globale demeure marqué par une forte domination des publications américaines et une sous-représentation de chercheurs domiciliés à l'extérieur des Etats-Unis.

\section{L'émergence des théories critiques}

Avec l'émergence des théories critiques dans le champ de la théorie de la politique globale, un ensemble de postulats, de points de repère et de manières de faire fut contesté. De plus en plus de travaux s'intéressent à la façon dont les communautés de chercheurs ont constitué et constituent leurs objets d'études`. On se demande comment certaines préconceptions de la politique globale ont contribué à raréfier certains objets d'études (la place des femmes, des classes, des autochtones, des réfugiés, ainsi que la politique du développement et de l'immigration dans la politique globale), à mettre sur un piédestal certains énonciateurs, à en délégitimer d'autres, et surtout à (re)produire les relations de pouvoir entre Etats.

Dès 1976, avec "On Thinking About the Future of the World Order ", Robert Cox commença à prendre ses distances avec la manière de faire de la revue International Organization. Etudiant les impasses rencontrées par diverses théories de la modernisation et de la politique globale, Cox, bien avant les constructivistes, amorce une réflexion sur les modalités par lesquelles la théorie participe à la construction de la politique globale. Il faudra encore cinq années pour qu'il propose une remise en question plus en profondeur des habitus de ce champ. Le coup d'envoi de l'école néogramscienne est donné en 1981 avec la publication de "Social Forces, States and World Order : Beyond International Relations Theory ». En 1987, il publie Production, Power and World Order qui devint le premier ouvrage de référence de cette approche. En 1990, Stephen Gill s'inscrit fermement dans le sillon de Cox avec la publication de American Hegemony and the Trilateral Commission ${ }^{10}$. Il édite, en 1993, Gramsci, Historical Materialism and International Relations ${ }^{11}$ où plusieurs auteurs (Giovanni Arrighi, Craig Murphy, David Law, Kees van der Pils) convergent vers ce courant. Entre 1981 et 1990, les néogramsciens ont joué un rôle important non seulement dans le développement de leur propre courant, mais également en jetant certaines bases de réflexion 
ultérieurement reprises par un ensemble de courants (post-structuralistes, féministes) qui tentaient de problématiser des nouveaux objets de la politique globale.

Yoseph Lapid qualifia de postpositiviste cette période caractérisée par l'émergence de débats, de problèmes et d'approches dans le champ de la théorie de la politique globale qui contestent la façon dont les débats à propos des représentations de celle-ci ont été structurés, en particulier pendant la Guerre Froide $^{12}$. En l'absence de meilleures expressions, on qualifie souvent de postpositivistes plusieurs approches qui s'opposent à au moins une des positions métathéoriques associées au rationalisme cartésien, à l'épistémologie proposée par Kenneth Waltz, et aux interprétations trop restrictives du modèle de l'acteur rationnel.

De 1981 à 2001, le champ se diversifie en raison d'un ensemble de facteurs : le déclin et la renaissance de l'hégémonie américaine; la fin de la Guerre Froide ; l'émergence ${ }^{13}$ de nouveaux acteurs non étatiques; et les emprunts plus diversifiés des théories de la politique globale à d'autres domaines du savoir. Cette remise en question amena une redéfinition des dispositions ontologiques, épistémologiques et normatives de ce champ. Certaines thèses défendues par les néogramsciens étaient déjà en circulation dans d'autres domaines de la théorie politique ${ }^{14}$. L'originalité de la position de Cox a été de critiquer Waltz sur son propre terrain, et de parvenir à présenter une position par rapport à laquelle les néoréalistes allaient devoir se positionner. Dans ce qui suit, nous présenterons, en centrant notre analyse autour de Cox et Gill, les positions ontologiques, épistémologiques et normatives néogramsciennes qui participèrent au développement des théories critiques de la politique globale ${ }^{15}$.

\section{Ontologie}

Deux éléments de l'ontologie de la politique globale retiendront notre attention, l'opposition entre parcimonie et complexité et l'opposition entre les positions ahistoriques et les critiques de la réification. Nous proposerons ensuite quelques exemples de la diversification de l'ontologie de la politique globale.

Sur le plan ontologique, peu de positions s'opposent davantage que celle de Kenneth Waltz, et de l'historien Marc Bloch de l'Ecole des Annales. Si le premier a longtemps tenu le haut du pavé dans le domaine de la théorie des Relations Internationales, c'est aujourd'hui par l'intermédiaire d'une série de recherches sur ses limites historiques que les fissures gagnent l'édifice du néoréalisme. Alors que le premier orientait son travail en fonction d'un souci de parcimonie, palpable dans toutes les étapes de sa démarche; le second, comme Braudel et Foucault, tient compte d'un ensemble d'éléments des plus diversifiés en portant une attention particulière à ce que l'histoire politique a longtemps considéré comme le moins politique et le plus anodin. Plus fidèles à la seconde de ces tendances, les théories critiques de la politique globale sont sceptiques face à l'engouement pour la parcimonie du néoréalisme : «L'élégance et la clarté de ses affirmations théoriques se font au prix d'un mode de compréhension peu convaincante de l'histoire $»^{16}$.

La parcimonie est parfois présentée comme un critère révélant la qualité d'une théorie scientifique. Il faut effectivement concéder aux défenseurs de celle-ci qu'ils partent d'une prémisse raisonnable. Puisqu'il est impossible de tracer un portrait empirique de l'ensemble de la politique globale, il faut procéder à son découpage au moyen de concepts et d'hypothèses générales (qu'on les appelle principes, lois, postulats, 
hypothèses théoriques, etc.), afin de dégager d'un amas de bruits et de fureurs, l'objet que l'on cherche à expliquer ou à comprendre. C'est dans cette veine que l'on a parfois soutenu qu'un des mérites du néoréalisme était sa simplicité qui serait au fondement de sa grande portée explicative. Cette association supposée entre simplicité et scientificité est toutefois loin de faire ses preuves en philosophie des sciences ${ }^{17}$. Plusieurs énoncés pseudo-scientifiques ont justement comme trait distinctif d'être formulés à un niveau d'abstraction suffisamment élevé pour s'avérer toujours valables pour qui veut bien se donner la peine de trouver les signes de leur validité. Outre des fondements épistémologiques brumeux, l'appel à la parcimonie a une importante implication normative en délimitant quels objets devraient rester dans la marge du champ.

A l'exception des variantes relativistes du poststructuralisme, ce que les théories critiques remettent en question, ce n'est pas le fait que certaines variables puissent avoir une plus grande portée explicative que d'autres, c'est le postulat selon lequel la sélection et la hiérarchisation des variables puissent être effectuées sur une base strictement cognitive et que celle-ci n'implique aucun choix normatif sur la nature de ce qui fait partie du champ de la politique globale et de ce qui en est exclu. Elles doutent qu'il soit possible de parvenir à une hiérarchie de variables formant une représentation consensuelle de la politique globale, et cela en dehors d'une entente préalable sur un arrière-plan normatif et politique devant guider la recherche. Pour cette raison, on peut résumer une première thèse des théories critiques: aucune ontologie de la politique globale n'est exempte d'une vision politique de celle-ci.

Les néogramsciens se méfient autant des théories qui postulent le caractère permanent du monde social, que de celles qui ne s'intéressent pas aux conditions d'émergence sociohistoriques et sociolinguistiques de leurs concepts. Il n'y a pas de connaissances complexes du monde social qui ne tiennent compte de l'étude des pratiques quotidiennes et des luttes menées par des forces sociales au sein de relations sociales concrètes.

A l'encontre de certaines perspectives constructivistes, Cox et Gill soutiennent qu'il n'est pas suffisant de présenter les faits sociaux et les normes (l'anarchie, la realpolitik, etc.) comme le fruit de constructions sociales. S'arrêter à ce constat revient à faire avorter l'analyse politique avant même qu'elle ne commence. Ce qui est réellement déterminant sur le plan politique, c'est le fait que ces faits sociaux et ces normes soient le produit d'une histoire, dont la dynamique principale est celle de la lutte entre des forces sociales. Les processus et les forces sociales qui dominent un ordre mondial donné ne sont pas seulement le résultat de constructions sociales; elles résultent des conflits entre les forces sociales d'une époque antérieure. La théorie néogramscienne est donc susceptible de se moudre aux transformations de l'ordre mondial au sein duquel elles évoluent.

\section{Epistémologie}

Sur le plan épistémologique, on se doit de dépasser certaines dichotomies qui, à ce stade, embrouillent les débats davantage qu'elles ne les éclairent. Les oppositions de Keohane entre les approches rationalistes et réflectivistes ${ }^{18}$, de Smith entre positivisme et post-positivisme, ou de Cox entre problem solving theories et critical theory ne semblent plus aptes à organiser les différents axes de débats des théories de la politique globale 
contemporaines. Formulés à travers ces dichotomies, les débats épistémologiques se sont avérés fertiles lors d'une période où le champ était particulièrement sclérosé, mais la réflexion sur ces questions a par la suite échappé à ces dichotomies pour être débattue en fonction de critères plus précis.

L'axe positivisme/post-positivisme en est un qui obscurcit aujourd'hui davantage qu'il n'éclaire les débats. Steve Smith décortique la position positiviste, qu'il associe aux théories traditionnelles des Relations Internationales, en fonction de quatre postulats : une "certaine unité (ontologique et/ou méthodologique) de la science»; la « distinction claire entre les faits et les valeurs »; l'existence de "régularités similaires dans les mondes naturels et sociaux»; "la démarche scientifique doit adopter une épistémologie empiriste» ${ }^{19}$. Cette présentation du positivisme ne satisfera sans doute pas tout le monde, mais elle a l'intérêt d'attirer l'attention sur des idées précises. Afin de situer les thèses des théories critiques par rapport au néoréalisme, nous rappellerons certains principes de ce dernier. Si l'on se fie aux critères de Smith, même Waltz ne peut pas être rigoureusement qualifié de positiviste. Tout en demeurant dans une tradition qui se veut scientifique, elle est moins empiriste que celle des béhavioristes. Elle critique l'emploi de l'induction dans les programmes de recherche auxquels s'oppose le néoréalisme. Selon Waltz, non seulement les faits ne parlent pas d'eux-mêmes, mais ils n'ont aucun sens en dehors d'une théorie. Il soutient que la théorie n'est pas quantitativement différente, mais qualitativement différente de la stricte observation de faits. Il s'oppose aux inductivistes selon lesquels il suffirait "d'accumuler des faits » pour bâtir une théorie, car la théorie n'a pas le même statut que « les faits». L'identification d'une corrélation entre certains événements ne peut pas se substituer à une explication de ceux-ci. Une théorie doit être constituée d'énoncés universels expliquant les liens entre des variables. Elle doit être construite en isolant, extrayant et agrégeant des variables. Et il faut vérifier la théorie en procédant par étapes ${ }^{20}$.

Pour Waltz, c'est la méthodologie et non l'ontologie qui doit unifier les sciences naturelles et sociales. C'est la capacité de prédiction d'une théorie qui est fondamentale pour comparer les théories entre elles. Dans la mesure du possible, une bonne théorie devrait permettre l'exercice d'un contrôle sur les événements ou phénomènes observables. Waltz s'inscrit à ce titre dans la tradition de Francis Bacon pour qui la nature devait être domestiquée et contrôlée. Dans cette tradition, l'arrière-plan normatif essentiel hérité du projet politique des Lumières est que l'autonomie des citoyens passe par leur capacité à domestiquer la nature, à se rendre maîtres de leur environnement et à se libérer de la vulnérabilité dont ils sont accablés au stade " précivilisé ». Ce sont certains éléments de cette tradition épistémologique qui firent l'objet d'une critique de l'Ecole de Francfort au XX $\mathrm{XX}^{\mathrm{e}}$ siècle. La thèse centrale de celle-ci est que le projet philosophique des Lumières, parce qu'il est centré autour du désir de parvenir à une maîtrise et à un contrôle des mondes naturel et social, contient dès l'origine les germes de sa régression. Contemporains de la soumission de l'art aux lois du marché, spectateurs de régimes politiques qui utilisent l'ingénierie sociale (social engineering) afin d'incarcérer, de contrôler et d'exterminer les masses, Adorno et Horkheimer dressent un bilan négatif du déploiement de la rationalité instrumentale. Celle-ci soumet l'ensemble des domaines de l'activité humaine à une logique comptable ne servant plus des fins émancipatrices, mais s'avérant au contraire une menace. 
Cependant, lorsque Adorno et Horkheimer se livrèrent à une critique du mode de pensée scientifique pendant le nazisme, c'était au nom de la raison elle-même : « [Si] la raison n'entreprend pas un travail de réflexion sur ce moment de régression, elle scellera son propre destin. (...) Ce qui est en cause, ce n'est pas la conservation du passé, mais la réalisation des espoirs du passé $»^{21}$. L'héritage de la pensée des Lumières est alors revendiqué par des protagonistes qui ont des vues opposées de la relation entre le mode de pensée scientifique et l'émancipation humaine.

Plusieurs thèses épistémologiques mises en avant par les théories critiques de la politique globale s'inscrivent dans le sillon de l'Ecole de Francfort. La critique de l'essentialisme, du naturalisme et de la réification, centrale chez les néogramsciens, poststructuralistes et chez certaines féministes, figurait parmi les thèmes centraux de la Théorie Critique durant l'entre-deux-guerres ${ }^{22}$. Bien que Cox se réclame de Vico et de Gramsci davantage que de l'Ecole de Francfort, la critique qu'il adresse au scientisme du néoréalisme s'inscrit dans le même sillon des thèses de celle-ci. Cox qualifie le néoréalism de problem solving theory, c'est-à-dire une forme de théorie qui se contente d'isoler et de résoudre certains problèmes de la politique internationale. La Théorie Critique prend comme objet d'analyse les conditions d'apparition historique des relations de pouvoir d'un ordre mondial et expliquerait les événements de la politique globale à la lumière des contradictions qui résultent de cette structure globale et historique. Les problem solving theories ne sont donc pas inefficaces ou inappropriées, mais elles segmentent leur ontologie dans le temps et dans l'espace. De façon générale, cinq éléments font défaut aux problem solving theories : l'analyse des conditions d'émergence historique de certains problèmes politiques globaux; la présentation du caractère social et non naturel de ces problèmes; l'indication de la façon dont ces situations problématiques bénéficient à certaines forces sociales au détriment d'autres ; la présentation de ces problèmes au sein de la logique propre à un ordre mondial donné; toute mention des voies qui pourraient mener à la transformation ou à la révolution d'un ordre mondial, de façon à favoriser l'émancipation et l'autonomie d'un plus grand nombre d'individus en son $\operatorname{sein}^{23}$.

Les néogramsciens rejettent le dualisme cartésien entre le sujet connaissant et son objet d'étude. Le processus de production intellectuelle n'est pas neutre, mais est issu de pratiques sociales qui participent au renforcement ou à la critique de relations de pouvoir. L'idée selon laquelle la conscience humaine participe à la transformation du monde social est une thèse centrale de la théorie critique depuis Hegel. Partagée autant par les hégéliens que par certains marxistes, elle est reprise par un ensemble de théories aujourd'hui qualifiées de constitutives parce qu'elles soutiennent que le chercheur joue un rôle dans la production-interprétation d'une vision du monde. Selon les néogramsciens, la connaissance intelligible du monde social n'est possible "que comme une création de l'esprit humain $»^{24}$. Le monde social en tant qu'objet de connaissance doit être appréhendé comme le résultat de pratiques sociales dont le sens est partagé de façon intersubjective et doit être constamment redéfini en fonction des transformations des pratiques sociales et de l'ordre mondial. Sur ce point, les théories critiques rompent avec une conception du sujet connaissant associée au positivisme. En opposition au cartésianisme, à l'empirisme anglais, et à l'idéalisme kantien, elles cherchent à situer le sujet connaissant dans son contexte social et historique, et à comprendre comment les conditions sociales (classe, "race», genre, statut) qui ont participé à la formation de sa subjectivité influencent la façon dont il questionne et analyse son objet de connaissance. Elles conçoivent la pensée, les concepts et les 
normes comme le produit d'un contexte historique largement déterminé par le mode et les relations de production. La théorie contribue donc à la production du monde social, et est en mesure de le modifier.

Les théories critiques rompent également avec les catégories normatives qu'elles attribuent à la théorie traditionnelle : l'utilité, la finalité rationnelle, et la productivité. Dans un monde qu'ils décrivaient durant le nazisme comme barbare, Adorno et Horkheimer attribuèrent à la théorie le rôle de négatrice de la totalité donnée, au sein de cette totalité, l'attitude critique ne peut promouvoir que «la modification de l'ensemble du système social $\aleph^{25}$. Selon ces deux auteurs, la théorie traditionnelle promeut une conception de la raison qui se met au service du capital et de la technique. Elle devient calculatrice et s'interdit tout jugement moral. La raison, d'abord prisée comme un outil permettant l'accroissement de l'autonomie individuelle en favorisant, selon Kant, "la sortie de l'homme de sa minorité ", devint instrumentale. Elle serait devenue stérile et condamnée à contempler son autodestruction.

Cette problématique est fondamentale pour la théorie de la politique globale. De plus en plus on pose la question des finalités poursuivies par les théories de la politique globale, ceci autant dans le domaine de l'économie politique que de la sécurité. Les institutions internationales interviennent constamment dans un ensemble d'Etats dans l'intention d'y favoriser le développement économique. Or, souvent ces programmes ont un impact non seulement économique, mais aussi politique et culturel. Ils ne remettent pas seulement en question l'organisation nationale de la production, mais également le rôle de l'Etat, de la société civile ${ }^{26}$, de l'organisation de la communauté et de la famille ${ }^{27}$. L'économie politique des relations internationales et les théories du développement sont donc beaucoup plus qu'une branche de la science économique, elles soulèvent également des enjeux qui relèvent du politique et de l'éthique. Trop souvent l'implantation de ces programmes est considérée d'un point de vue technique, elle vise l'application d'un savoir instrumental et apolitique. Dans le domaine de la sécurité, ce n'est pas l'application d'un savoir instrumental sur le développement économique qui dépolitise et technicise les processus politiques, mais le processus de sécurisation via lequel des enjeux sont retirés de l'espace public par les professionnels de la sécurité et les gestionnaires de la menace ${ }^{28}$.

Cela nous mène à reposer la question de la division entre les jugements de fait et les jugements de valeur. Peut-on théoriser la politique globale en dehors d'une certaine conception normative de celle-ci ? Selon Smith, les positivistes défendent l'idée qu'il est possible de séparer ces deux questions : qu'en est-il des théories critiques? Cette question est délicate. Lorsque l'on nie la capacité de séparer les jugements de fait et les jugements de valeur, il est important de préciser si on le fait au niveau des énoncés d'observation ou des énoncés théoriques. Les théories critiques ne nient pas qu'un énoncé d'observation puisse constituer un fait déductible en fonction des postulats que se donne une théorie quelconque. Cox par exemple remet en question la neutralité politique des théories, sans nier toutefois la valeur heuristique des problem solving theories. Il soutient cependant que la théorie, en plus d'avoir une valeur explicative, peut renforcer, mettre en évidence, voire ébranler des relations de pouvoir ${ }^{29}$. Ce que les néogramsciens nient c'est que les énoncés théoriques d'une théorie de la politique globale soient complètement exempts de jugements de valeur. Sans certains énoncés normatifs sur ce qu'est la sécurité et sur ce qui doit être sécurisé, il est impossible d'établir ce qui constitue ou non une menace à la sécurité. Pour Cox, ce qui distingue les théories critiques c'est le fait qu'elles fassent la genèse historique de la consolidation 
des relations de pouvoir, qu'elles en indique le caractère social, et qu'elles visent à émanciper les individus des relations de pouvoir.

Cette présentation sommaire devrait commencer à suggérer les raisons pour lesquelles l'invitation de certains néoréalistes et néolibéraux à comparer les théories rivales en fonction de leur capacité de prédiction ${ }^{30}$ revenait à imposer un critère important pour certains, mais secondaire pour d'autres. De plus, les chercheurs du camp "néo-néo » ont intérêt à faire acte de modestie en matière de prédiction. Ils furent incapables de prédire la plus importante transformation du système international des vingt dernières années : l'effondrement du Bloc de l'Est en 1989. L'ontologie « néo-néo » en négligeant l'étude des relations intra-étatiques est par ailleurs incapable de rendre compte des transformations sociales et du repositionnement des forces sociales qui précipitèrent l'effondrement de l'ancien Bloc de l'Est. Ceci sans parler de son incapacité à prédire l'émergence spectaculaire de nouvelles formes de résistances non étatiques au sein de la politique globale. Devant des acteurs prêts à se transformer en bombes humaines, les explications du comportement reposant sur la fonction d'utilité font piètres figures.

\section{Teoria cui bono?}

Le champ de la théorie de la politique globale, n'a pas été épargné par une variante du postmodernisme prônant l'impossibilité de fonder les théories politiques autant sur le plan théorique que normatif. Jim George par exemple consacre trois chapitres de son Discourses of Global Politics: A Critical (Re) Introduction to International Relations à disserter sur son ambivalence à l'égard de la Théorie Critique et de la déconstruction. Cox quant à lui, résume bien la position des néogramsciens en affirmant, à propos de la déconstruction, la « tâche de défricher le terrain ne devrait pas devenir un obstacle à la construction d'une nouvelle perspective, même si celle-ci s'avère à son tour susceptible de subir une réévaluation critique $»^{31}$.

A la fin des années 1970, Habermas interrogeait les différentes sciences en fonction du type d'intérêt qui y oriente la recherche. La thèse qu'il défendait à l'époque n'étaient qu'une partie des sciences sociales (praxéologique), dont l'intérêt principal devrait être l'émancipation des individus et l'accroissement de leur autonomie, était trop souvent orientée en fonction d'un intérêt de connaissance qui devrait être le propre des sciences de la nature (i.e. l'acquisition d'un savoir technique à propos du monde social visant davantage le contrôle que l'émancipation de la société). C'est dans cette mouvance que se situent des positions qui mirent au centre de leur théorie de la politique globale la réduction des contraintes exercées sur l'autonomie des citoyens de la Polis globale ${ }^{32}$. Ainsi, selon Neufeld «si on doit faire une contribution valable, une transformation fondamentale de la discipline - il faut une 'restructuration' de la discipline qui mettra la question de l'émancipation humaine en première place $»^{33}$.

Pour Neufeld, l'émancipation et la normativité émergèrent comme problématiques importantes de la théorie de la politique globale lors du «troisième débat ». A cette occasion, entre 1985 et 1995, la problématique de la réflexivité devint centrale pour les théories critiques. Dans ce débat qui opposa néoréalistes, libéraux et " néomarxistes ", chaque approche resta généralement campée sur sa position. Face à ce cloisonnement et au refus de prendre les alternatives au sérieux, un premier point de vue émergea selon lequel les différentes approches sont incommensurables et donc incomparables. Selon cette vision de la théorie, les approches divergent à un point tel qu'elles doivent 
évoluer en vase clos, car il manque de standards en fonction desquels on pourrait les comparer. Selon d'autres, la reconnaissance d'une incommensurabilité entre ces approches implique que le débat entre celles-ci doit se poursuivre au niveau des présupposés normatifs des théories rivales. L'attention des défenseurs de cette seconde option se déplace donc au niveau des présupposés métathéoriques. C'est dans ce contexte que le concept de réflexivité devient central ${ }^{34}$. La réflexivité, qui émergea d'une insatisfaction devant les niveaux d'analyse proposés par les modèles des années 1980, trouve une première formulation articulée en théorie de la politique globale chez Neufeld ${ }^{35}$. La réflexivité s'intéresse aux présupposés normatifs d'une théorie et à la vision du monde qu'elle véhicule et tend à (re)produire, et se caractérise par deux thèses. La première porte sur la relation entre la théorie et la pratique, selon Neufeld, "de même que les faits et les valeurs, les communautés de recherches et les agendas politiques sont toujours imbriqués $»^{36}$. Autant sur le plan conceptuel, que sur celui de la démarche de recherche, la réflexivité remet en question la division entre théorie et pratique. Selon la seconde thèse, il est possible et nécessaire de "s'engager dans un dialogue rationnel à propos du mérite respectif des paradigmes rivaux ${ }^{37}$. Les approches rivales doivent être comparées en fonction de la place qu'elles accordent à l'émancipation et à l'autonomie des individus au sein de leur vision de la politique globale. La réflexivité n'est donc pas une approche théorique, mais une disposition à s'engager dans un débat ouvert et rationnel à propos des enjeux normatifs et métathéoriques inhérents au processus de théorisation.

Neufeld proposa trois critères que devrait remplir une approche réflexive. Premièrement, elle doit établir clairement les prémisses sur lesquelles repose la construction d'une théorie : un chercheur doit "porter attention, et divulguer les présuppositions trop souvent non mentionnées sur lesquelles sont érigés les édifices théoriques». Ensuite il lui faut la reconnaissance par les chercheurs "du contenu politico-normatif des paradigmes et de la tradition de science normale qu'ils génèrent ». Finalement, Neufeld invite les réflexivistes à surpasser l'anxiété cartésienne selon laquelle il serait impossible d'avoir « des jugements raisonnés en l'absence d'un langage observationnel neutre $\aleph^{38}$. De façon générale, les théories critiques de la politique globale adoptent une des trois positions normatives suivantes : ou bien elles tentent de montrer comment des relations de domination trouvent leur fondement dans l'organisation des relations sociales de production; ou bien elles situent les conditions d'émergence historiques d'entités ou de processus considérés comme naturels par les théories traditionnelles; ou, enfin, elles cherchent à favoriser le développement d'une communauté globale où le plus possible d'acteurs pourraient se faire entendre sur une base légitime et rationnelle.

Dans cette veine, néomarxistes et néogramsciens soutiennent que la théorie de la politique globale doit porter attention à des groupes sociaux affectés par les conséquences des phénomènes globaux, mais systématiquement laissés pour compte par les théories traditionnelles. Alors que les poststructuralistes développent cette critique en attirant l'attention davantage sur les groupes exclus, ou les plus mobilisés au nom de l'intérêt national. 


\section{Quelques axes de recherches sur la production théorique de l'insécurité}

Un des apports de Gramsci à la tradition du marxisme occidental est sa thèse selon laquelle le pouvoir de la classe dominante ne repose pas seulement sur la coercition, mais également sur sa capacité à étendre son idéologie à travers un ensemble d'institutions de la société civile (l'église, le système d'éducation, etc.). La classe dominante acquiert un pouvoir hégémonique sur la société civile, lorsque les principales institutions de celle-ci ne contestent pas son pouvoir et prennent sa défense, ou du moins la défense de « l'ordre ». Selon Gramsci, une des raisons de l'échec des marxistes italiens à s'emparer du pouvoir fut leur incapacité à instaurer un bloc contre-hégémonique au sein de la société civile italienne.

Les néogramsciens critiquent le néoréalisme, mais se veulent également une alternative aux approches marxistes qu'ils qualifient d'orthodoxes. Ils s'inspirent autant du marxisme occidental (Gramsci, Thompson, Anderson) que d'auteurs qui s'intéressent moins à l'analyse de classe (Braudel, Polanyi, Wallerstein) ${ }^{39}$. Les travaux récents de Gill sur le disciplinary neoliberalism s'inspirent également de Foucault. Une des hypothèses théoriques principales des néogramsciens est qu'il faut considérer « les relations de pouvoir au sein des sociétés et de la politique globale sous l'angle des relations de pouvoir reliées à la production $»^{40}$. Ce qui sous-tend leurs analyses c'est « la prémisse selon laquelle le travail est une activité fondamentale qui affecte un éventail d'autres relations sociales et l'organisation de la société dans son ensemble $»^{41}$. Gill rappelle que "pour Gramsci, c'est l'ensemble des relations sociales configuré par les structures sociales (la situation) qui est l'unité d'analyse de base $»^{42}$. Ainsi, la politique globale forme un tout cohérent dont la dynamique structurante est l'expansion modernisatrice du mode de production capitaliste. L'étude des forces sociales, des structures de production ou de la structure politique des sociétés civiles ne peut donc pas faire abstraction de l'ensemble des forces matérielles et des relations sociales qui influencent les dynamiques de pouvoir au sein d'un ordre mondial.

Cox reprend les concepts de Gramsci en les appliquant au niveau de la politique globale. Selon lui, chaque ordre mondial se caractérise par une structure d'accumulation spécifique. Celle-ci correspond à une organisation et à une hiérarchisation des modes de relations sociales de production à travers lesquelles des surplus sont transférés de la périphérie vers le centre. Chez Cox, la relation centre-périphérie désigne une relation économique davantage que géographique. La structure d'accumulation d'un ordre mondial donné conforte la position de pouvoir d'un bloc historique. C'est-à-dire qu'un certain ensemble de forces sociales, de classes, d'Etats et de firmes en bénéficient au détriment d'autres. Un bloc historique repose toujours sur un équilibre flexible entre le consensus et la coercition. Afin de rester dominante, la puissance hégémonique assure sa légitimation auprès de ses alliés en les faisant bénéficier des retombés économiques et politiques de sa domination. Une partie du travail des néogramsciens se consacre à l'étude de la formation, de la consolidation, de la transformation et de la succession des blocs historiques. Chaque période hégémonique est étudiée en fonction de forces sociales qu'elle favorise et défavorise. Sur le plan normatif, l'approche néogramscienne se caractérise par sa volonté d'articuler des formes de résistance au sein des sociétés civiles locales, nationales et globale de façon à promouvoir une transformation émancipatrice des relations de pouvoir et de production. 
Néogramsciens et néomarxistes ne nient pas l'importance des processus sociolinguistiques dans la formation et la reproduction des relations de pouvoir, mais ils cherchent à montrer comment ils sont liés à des luttes de classes, à des tensions dans les relations sociales, à des conflits pour l'établissement d'une hégémonie; et, de façon générale, à identifier les conditions matérielles de la diffusion des discours et des représentations sociales. L'analyse du discours, des représentations sociales ou de l'identité collective qui ne prend pas en compte l'analyse des conditions matérielles de leur énonciation et de leur diffusion est aussi utile à la théorie de la politique globale qu'une bicyclette à un poisson. Il faut montrer le caractère socialement construit de certains éléments du monde social, mais une fois cette étape de l'analyse dépassée, il faut faire une économie politique de la mise en récit d'entités réifiées par la langue politique.

\section{Les Théories Critiques et l'émancipation}

Andrew Linklater est un des principaux importateurs de la Théorie Critique au sein des théories de la politique globale ${ }^{43}$. Il s'en inspire, en outre, lorsqu'il reproche aux marxistes de réduire la question de l'émancipation à celle de la lutte des classes. Comme Habermas et Gramsci, Linklater reconnaît une agence potentiellement émancipatrice à un ensemble d'entités en dehors des classes sociales. Il attribue essentiellement quatre idées fondamentales au vaste courant qu'il qualifie de Théorie Critique inspirée du marxisme en Relations Internationales : la thèse épistémologique de Habermas et de Cox selon laquelle le savoir reflète des formes d'intérêts préscientifiques; une thèse ontologique qui se veut une double critique de la réification, une critique de la tendance de certaines théories à considérer les structures de domination d'une époque donnée comme transhistoriques, et une critique des théories qui n'articulent pas le potentiel émancipatoire d'une époque donnée en fonction de la spécificité de ses structures sociales ; la Théorie Critique, contrairement au marxisme, attribue un potentiel émancipatoire à un ensemble de forces sociales réprimées, et cela que leur sort résulte ou non du processus de production; la Théorie Critique inspirée de Habermas fait résider le cœur de sa critique non pas dans l'analyse des relations sociales de production, mais dans le degré d'ouverture de l'espace public, et dans la capacité du plus grand nombre non seulement à se faire entendre, mais à participer démocratiquement au processus de décision politique ${ }^{44}$. Sur le plan international, cela se traduit par une réflexion sur le lien collectif et sur les conceptions de l'identité collective en mesure d'établir des communautés postnationales visant la réduction de l'exclusion et des rapports de domination.

Ken Booth s'inspire également de certains thèmes de la Théorie Critique afin de réformer les études traditionnelles de la sécurité ${ }^{45}$. Selon lui, les études de la sécurité doivent renouveler entièrement leur vocabulaire théorique et repenser les objets qu'elles cherchent à « sécuriser ». Dans cet ordre d'idées, l'émancipation des individus, bien plus que la reproduction de l'appareil étatique, devrait être au cœur des études de la sécurité. Selon Booth, le fait que les citoyens de plusieurs Etats aient plus à craindre de leur propre Etat que des Etats voisins rend caduque la limitation de la réflexion sur la sécurité à la «sécurité nationale ». Dans plusieurs Etats du Cône sud de l'Amérique latine c'est précisément la doctrine de la sécurité nationale qui vint justifier une succession de coups d'Etat militaire, d'enlèvements politiques et la torture 
systématique de l'opposition politique. Donnelly rappelle que les doctrines de la sécurité nationale furent fortement influencées par les théories contre insurrectionnelles, mises sur pied en outre par les Français dans les années 1950-60 afin de mater les soulèvements dans leurs colonies. C'est au nom d'une telle doctrine qu'en 1976, Iberico Saint Jean, gouverneur militaire à Buenos Aires, pouvait présenter son plan d'action la conscience tranquille : «[D'] abord nous tuerons tous les subversifs ; puis nous tuerons tous leurs collaborateurs; puis... leurs sympathisants; puis... les indifférents ; et enfin, nous tuerons les timides $»^{46}$. Très rassurant pour une doctrine de la sécurité... En faisant l'historique de ces doctrines de la sécurité, il apparaît que bien souvent elles ont plus à cœur de sécuriser les rapports de domination aux niveaux national et global, que la sécurité des populations civiles. C'est dans cet ordre d'idées que Booth refuse d'articuler sa conception de la sécurité aux concepts de pouvoir et d'ordre qui ont trop servi, d'une part, à sécuriser certains individus, groupes ou classes, au détriment de la sécurité d'autres; et d'autre part, à retirer des luttes sociales de l'espace public, afin de les relayer aux autorités en charge de défendre l'«intérêt national ».

\section{Réflexions sur l'ordre mondial contemporain}

En conclusion, il est important de rappeler que pour plusieurs théories critiques, s'opposant sur ce point à la philosophie politique, toute réflexion sur l'émancipation se doit d'être ancrée au sein d'un ordre mondial donné. Pour la Théorie Critique, il n'y a pas d'émancipation en général. Les pratiques sociales plus favorables à l'émancipation d'un groupe donné sont toujours inscrites dans un contexte social spécifique structuré par une marge de manœuvre restreinte. Dans chaque contexte social, certaines pratiques sociales auront pour effet d'accroître l'autonomie d'un plus grand nombre d'individus ou groupes, alors que d'autres auront tendance à en favoriser une minorité. Un des défis des théories critiques de la politique globale est de ne pas perdre de vue les liens entre les dynamiques locale, nationale, régionale et globale qui constituent la politique globale contemporaine.

Par les temps qui courent, il faut se méfier des analystes de la politique globale qui sont convaincus d'avoir raison. Depuis le 11 septembre 2001, les théories critiques se trouvent dans une position fragile et les limites du possible sont restreintes. Elles font face à un nouveau contexte par rapport auquel elles doivent se situer. Ce contexte est, entre autres, marqué par une polarisation accrue des débats sur l'hégémonie américaine, dans un contexte de production intellectuelle où la dissidence est moins prisée que le salut au drapeau. Quoi qu'il en soit, les opposants à la domination américaine sont nombreux autant dans le champ académique, que sur la scène politique. La conjoncture politique depuis le 11 septembre 2001 est suffisamment sérieuse pour que les théories critiques fassent le tri entre les critiques de l'hégémonie américaine qui ont une portée émancipatrice, et celles qui relèvent de projets politiques conservateurs. L'échec au Nord des partis politiques sociaux-démocrates et socialistes à résister au vent néo-conservateur durant les vingt dernières années a favorisé l'essor de partis néo-populistes, surtout dans les Etats dotés d'un système politique bipartiste. Ces partis défendent une plate-forme culturelle ultraconservatrice, et se rallient des segments de la population traditionnellement fidèles à l'électorat de la gauche. Au Sud, l'élimination des forces d'oppositions socialistes a 
débouché en outre sur une "islamisation» des foyers de résistance anti-américains. L'effet de blow back face à la politique étrangère américaine dans la périphérie est canalisé non plus par les mouvements socialistes, mais par des segments minoritaires du monde islamique dont certains étaient les alliés des EU lors de la Guerre Froide. C'est ainsi que les enfants de Huntington voient le jour.

Paradoxalement, l'émergence de ces forces néoconservatrices au Nord et au Sud a tendance à se conforter. Au Nord, le discours des professionnels de la sécurité sur la «menace terroriste» consolide le soutien au programme des forces sociales néoconservatrices (politique anti-immigration, augmentation des budgets de la sécurité intérieure); alors qu'au Sud, l'hypocrisie du discours de l'administration Bush, qui combat à la fois le "terrorisme " et la Cour pénale internationale, décourage les militants des droits de l'homme et les forces sociales en faveur de l'établissement d'un Etat de droit global. Les premiers à bénéficier de l'unilatéralisme américain et, de son opposition à l'établissement de la CPI, sont ceux qui s'opposent à la règle du droit et prônent une guerre tout azimut à l'Occident.

Les théories critiques de la politique globale se retrouvent donc retranchées dans une position où elles ne peuvent pas être complaisantes avec les défenseurs de l'hégémonie américaine, ni avec ceux qui réifient le monde occidental en une entité homogène à abattre. Elles ont maintenant pour tâche de montrer comment se sont constitués historiquement les tensions et conflits contemporains aux niveaux global, national et local en montrant qui en a bénéficié. Elles doivent indiquer en quoi les contradictions économiques et politiques du monde contemporain relèvent d'un ordre mondial spécifique, et finalement elles doivent désigner les forces sociales qui militent encore en vue de l'établissement d'une polis globale. Une des difficultés que rencontrent les théories critiques est celle de théoriser la création d'un front commun d'une part face à l'hyperlibéralisme, et d'autres part, face au nihilisme postmoderne. Dès 1991, Cox diagnostiquait cette faiblesse des mouvements sociaux qui militent à l'échelle globale.

$\mathrm{Au}$ cœur du défi se trouve la question de la force motrice du changement. Un des effets de la mondialisation de la production est que l'on peut plus parler de façon sérieuse de la «classe ouvrière » comme une force sociale unifiée au niveau national, et encore moins au niveau international. La classe ouvrière connaît aujourd'hui une existence objective fragmentée et une conscience commune très problématique. Dans la nouvelle structure sociale émergente, des catégories qui définissent seulement en rapport avec la production sont compliquées par les catégories de genre, ethnicité, religion et région, puisque celles-ci constituent souvent la base de la segmentation. Ces catégories ne remplacent pas la production comme le facteur principal qui structure la société. Elles deviennent des facteurs intermédiaires, la base de la conscience de soi des identités de groupe, entre relations de production et forces sociales ${ }^{47}$.

Ce diagnostic présentait clairement ce qui oppose les néogramsciens à certains postmodernes. La question centrale pour les néogramsciens demeure celle de l'émancipation face à des mécanismes de domination et d'exploitation qui ont des racines essentiellement économiques. Bien souvent, cette domination est vécue phénoménologiquement à un autre niveau. C'est-à-dire qu'elle est vécue, intériorisée, puis traduite, au niveau du genre, de l' " ethnie ", de la nation, de la religion, etc. Les néogramsciens ne nient pas que des relations de pouvoir existent également à ces niveaux. Cependant, ils voient dans cette segmentation des dominés, un obstacle à la 
formation d'un bloc contre-hégémonique en mesure de contrer les effets de l'hyperlibéralisme ${ }^{48}$.

Les débats en amont de la publication de Empire de Hardt et Negri ${ }^{49}$ soulignent que des désaccords surgissent quand vient le temps d'identifier les sources des maux de la politique globale contemporaine. Cox et Gill prennent part à cette réflexion ${ }^{50}$. Sur un point, les néogramsciens s'accordent avec les auteurs de Empire pour soutenir l'idée que pointer dans la seule direction des Etats-Unis pour décrire les assises matérielles et idéologiques de la structure de pouvoir actuelle serait sous-estimer la nature diffuse de celle-ci. Là s'arrêtent les rapprochements. Selon les néogramsciens, il demeure fondamental de faire une économie politique de cette structure, d'identifier les forces sociales qui en bénéficient et de leur opposer une résistance à partir de la société civile, globale dans la mesure du possible.

C'est en outre cette difficulté à mettre le doigt sur ce qui se cache derrière l'Empire qui rend difficile l'articulation de résistances à celui-ci. Sur ce point, la démarche des critiques de cet ouvrage, surtout celle de Cox, demeure hésitante. En quelque sorte, c'est tant mieux. On aurait de bonnes raisons de se méfier d'un abécédaire de la résistance. Cette hésitation tient au fait qu'ils tentent de tirer bonne note de la leçon postmoderne en articulant un projet de résistance qui soit le plus inclusif possible, mais sans se satisfaire de l'invitation évasive de Hardt et Negri à déconstruire l'Empire à partir du potentiel créateur de la multitude. Cox rappelle que « pour qu'une alternative à la globalisation devienne viable, elle devra transcender les multiples divisions basées sur l'ethnicité, le genre, la religion et la géographie $»^{51}$.

L'appel des Cox et Gill en faveur de la résistance à partir de la société civile a un avantage stratégique et un inconvénient théorique. Face au matérialisme historique, son avantage stratégique est son inclusivité. Effectivement, il est plus difficile d'utiliser le concept de classe aujourd'hui qu'à la fin du XIX ${ }^{\text {ème }}$ siècle. Sur le plan théorique cependant, Cox rejette un peu rapidement la critique adressée par Ellen M. Wood aux théories de la société civile ${ }^{52}$. Celle-ci rappelle, que le concept de classe présente une contradiction fondamentale dans les relations sociales. La classe n'est pas seulement un lieu potentiel de résistance, mais également un outil dynamique d'analyse et d'action politique. Si Cox veut faire jouer à la culture et à la société civile le rôle qui était attribué aux classes dans le matérialisme historique, plus d'explications seront les bienvenues. Gramsci en formulant sa théorie de la société civile et de l'idéologie n'a jamais mis de côté l'analyse des relations de propriétés. C'est dans l'abandon de cette dimension que certains poststructuralistes et certains libéraux convergent sur le plan politique.

Le travail théorique sera crucial pour réagir rapidement à l'évolution de la politique globale, pour en effectuer une interprétation appropriée, aussi éphémère soit-elle, et pour se positionner devant les nouvelles contradictions et les nouveaux conflits qu'elle engendre. La conservation du potentiel émancipateur de la tradition occidentale dépendra encore une fois de la forme que prendront les conflits en son sein. 


\section{NOTES}

1. A l'instar de Robert Cox nous utiliserons l'expression «politique globale » davantage que celle de «relations internationales » pour trois raisons : elle caractérise mieux l'élargissement et la diversification du champ qui commence à s'effectuer au milieu des années 1980 ; l'expression « relations internationales » correspond essentiellement à la façon dont réalistes et idéalistes ont mis en récit l'histoire du champ où ils intervenaient et accorde une priorité ontologique aux Etats-nations qui nous semble injustifiée ; finalement, l'expression "politique globale » reflète mieux le point de convergence de plus en plus important entre la politique comparée, la théorie politique et l'étude des relations politiques, économiques, écologiques et de genre qui dépassent largement le cadre de l'Etat-nation. Cox R.W., «Toward a posthegemonic conceptualization of world order : reflection on the relevancy of Ibn Khaldun », in Cox R.W., T.J. Sinclair, Approaches to World Order, Cambridge, Cambridge University Press, 1996, p. 144.

2. Voir Bigo D., «Grands débats dans un petit monde : Les débats en relations internationales et leur lien avec le monde de la sécurité », Cultures \& Conflits, 19-20, 1995, pp.7-49.

3. Bourdieu P., Langage et pouvoir symbolique, Paris, Editions Fayard, 2001, p. 187.

4. Cox R.W., "Social Forces, States and World Order : Beyond International Relations Theory ", Millennium, 10, 2, 1981, pp. 126-155.

5. Fukuyama F., La fin de l'histoire et le dernier homme, Paris, Flammarion, 1992.

6. Voir Eagleton T., Ideology, London, Verso, 1991.

7. Voir Habermas J., Droit et démocratie. Entre faits et normes, Paris, Editions Gallimard, 1997.

8. Voir Bourdieu P., Science de la science et réflexivité, Paris, Editions Raisons d'agir, 2001.

9. Voir, entre autres, Cox R.W., «On Thinking About the Future of World Order », World Politics, 28, 1976, pp. 175-196 ; Bigo D., op. cit. ; Cohn C., « Sex and Death in the Rational World of Defense Intellectuals », Signs, 14, 47, 1987, pp. 687-718; Cynthia Enloe, Bananas, Beaches and Bases : Making Feminist Sense of International Politics, $2^{\mathrm{e}}$ édition, Berkeley, Los Angeles et Londres, University of California Press, 2000.

10. Gill S., American Hegemony and the Trilateral Commission, Cambridge, Cambridge University Press, 1990.

11. Gill S., Gramsci, Historical Materialism and International Relations, Cambridge, Cambridge University Press, 1993.

12. Lapid Y., "The Third Debate : On the Prospect of International Theory in a Post-Positivist Era ", International Studies Quarterly, 33, 3, 1989, pp. 235-254.

13. Ce n'est pas tellement qu'ils émergent durant cette période, mais plutôt qu'ils ne peuvent plus être passés sous silence.

14. La théorie critique de l'Ecole de Francfort en particulier. Voir notamment Habermas J., La technique et la science comme idéologie, Paris, Editions Gallimard, 1968.

15. La division ontologie, épistémologie et normativité réfère à des distinctions qui se recoupent souvent. Nous présentons les théories critiques davantage comme une mouvance organisée autour d'un ensemble de thèses (qui font encore l'objet de discussion) et de manières d'aborder la politique globale, que comme un corpus cohérent et achevé.

16. Cox R.W., "Toward a posthegemonic conceptualization of world order : reflection on the relevancy of Ibn Khaldun », op. cit., p. 53. Toutes les traductions sont de l'auteur.

17. Voir Bunge M., The Myth of Simplicity, Englewood Cliffs, NJ : Prentice-Hall, 1963 ; et du même auteur, Social Science Under Debate, University of Toronto Press, 1999.

18. Keohane R.O., "International Institutions : Two Approaches ", International Studies Quarterly, 32, 4, 1988, pp. 379-396. 
19. Smith S., "Positivism and Beyond " in Smith S., Booth K., Zalewski M. (dir.), International Theory: Positivism and Beyond, Cambridge, Cambridge University Press, 1996, p. 16.

20. Waltz K., "Laws and Theories ", in Keohane R.O. (dir.), Neorealism and its Critics, New York, Columbia University Press, 1986, p. 42.

21. Adorno T. et M. Horkheimer, La dialectique de la raison, Paris, Editions Gallimard, 1974, pp. 15-16.

22. Nous utilisons des majuscules pour distinguer la Théorie Critique associée à l'Ecole de Francfort des théories critiques en général.

23. Voir Cox R.W., " Gramsci, Hegemony and International Relations : An Essay in Method », in Gill S., (dir.), Gramsci, Historical Materialism and International Relations, New York, Columbia University Press, 1993, pp. 49-50.

24. Cox R.W., " On Thinking About the Future of World Order », op. cit., p. 181.

25. Adorno T. et M. Horkheimer, op. cit., p. 39.

26. Bien souvent cette société civile est inexistante ou à un stade embryonnaire ; l'Etat assure une grande part de l'activité économique. A court terme, la déstructuration de l'Etat a davantage pour effet de favoriser la violence urbaine et la migration vers l'extérieur, que de consolider la société civile.

27. La famille nucléaire du capitalisme avancé est apparue à un stade très tardif de l'industrialisation. Elle a été le résultat et non pas le moteur d'un ensemble de développements économiques spécifiques à ces Etats. La famille élargie joue un rôle essentiel dans le mode de subsistance de plusieurs Etats qui n'ont pas subi ces transformations.

28. Voir Bigo D., op. cit.

29. Cox R.W., "Social Forces, States and World Order : Beyond International Relations Theory ", op. cit.

30. Notons que la capacité d'une théorie à faire des prédictions n'est pas un gage de scientificité. La théorie de la sélection naturelle explique beaucoup de choses, sans toutefois pouvoir en prédire avec assurance.

31. Cox R.W., "Toward a posthegemonic conceptualization of world order : reflection on the relevancy of Ibn Khaldun ", op. cit., p. 146.

32. Voir Linklater A., Beyond Realism and Marxism : Critical Theory and International Relations, New York : St Martin's Press, 1990 ; du même auteur, The Transformation of Political Community, Cambridge, Polity Press, 1998 ; "The achievements of critical theory », in Smith S., Booth K. et Zalewski M. (dir.), op. cit., pp. 279-298 ; et Neufeld M., The Restructuring of International Theory, Cambridge, Cambridge University Press, 1995.

33. Neufeld M., , op. cit., p. 13.

34. Ibid., pp. 49-69.

35. Neufeld M., « Reflexivity and Intnernational Relations Theory », Millennium, 22, 1, 1993.

36. Ibid., pp. 60-61

37. Ibid.

38. Ibid., pp. 55-58.

39. . En 1987, Cox se situa au sein du débat opposant les auteurs qui théorisent le capitalisme davantage au niveau de la sphère des échanges (Gunder Frank, Wallerstein) à ceux qui le théorisent au niveau de la sphère de la production (Anderson, Brenner, Wood). Il prit parti pour les seconds dans ce débat. Voir Cox R.W., Production, Power and World Order, Social Forces in the Making of History, New York, Columbia University Press, p. 51.

40. Ibid., p. 1

41. Ibid., p. ix.

42. Gill S., (dir.), Gramsci, Historical Materialism and International Relations, Cambridge : Cambridge University Press, p. 24. 
43. Voir notament les écrits suivants, Beyond Realism and Marxism : Critical Theory and International Relations, op. cit., The Transformation of Political Community, Cambridge, Polity Press, 1998, et « The achievements of critical theory ", op. cit.

44. Linklater A., "The achievements of critical theory », op. cit, pp. 279-280.

45. Booth K., " Security and Emancipation », Review of International Affairs, 17, 4, 1991, pp. 313-326.

46. Cité dans Jack Donnelly, International Human Rights, Boulder, Westview Press, 1998, p. 41

47. Cox R.W., "Toward a posthegemonic conceptualization of world order : reflection on the relevancy of Ibn Khaldun », op. cit., p192.

48. L'argument ici n'est pas que les formes de domination fondées sur le genre, l' « ethnie », ou la «nation », l'orientation sexuelle, sont des effets co-latéraux de la domination économique. Ces formes de domination et de discrimination ont leurs dynamiques propres qui peuvent être renforcées ou atténuées par des transformations dans les relations sociales de production. L'émancipation dans le domaine économique n'implique nullement que les autres formes de domination disparaîtront dans la même mouvance.

49. Hardt M. et A. Negri, Empire, Paris, Exils, 2000.

50. Voir Cox R.W., Schechter M.G., The Political Economy of a Plural World. Critical Reflections on Power, Morals and Civilization, Londres et New York, Routledge, 2002 ; Gill S., Power and Resistance in the New World Order, New York, Palgrave, 2003.

51. Cox R.W., The Political Economy of a Plural World. Critical Reflections on Power, Morals and Civilization, op. cit., p. 85.

52. Wood E.M., Democracy against Capitalism : renewing historical materialism, Cambridge, Cambridge University Press, 1995.

\section{RÉSUMÉS}

Cet article retrace les principales positions soutenues par des auteurs ayant contribués à l'analyse néogramscienne des relations internationales. Depuis les années 1980, l'analyse néogramscienne est un des cadres d'analyse principaux à s'être situé en réaction d'une part aux approches traditionnelles des Relations Internationales, et d'autre part aux théories marxistes orthodoxes. Ce cadre théorique, d'abord développé par Robert W. Cox en réponse aux insuffisances théoriques, et surtout à l'a-historicisme de l'analyse de Kenneth Waltz, fait aujourd'hui partie des théories incontournables des Relations Internationales. En outre, l'analyse néogramscienne a joué un rôle central dans la critique de l'influence positiviste et américanocentriste qui a été fortement en question avec le Troisième Débat en théorie des Relations Internationales.

This article looks back at the main positions defended by those authors who have contributed to the neo-Gramscian analysis of international relations. Since the 1980 s, neo-Gramscian analysis has been one of the main analytical frameworks to situate itself as a reaction against traditional approaches to International Relations, on the one hand, and against orthodox Marxist theories, on the other. This theoretical framework, first developed by Robert W. Cox in response to Kenneth Waltz's theoretical shortcomings, and especially his ahistoricism, has become one of the essential theories of International Relations. Moreover, neo-Gramscian analysis has played a central role in the critique of the Americano-centred, positivist influence which was vigourously questioned with the Third Debate in International Relations Theory. 
INDEX

Mots-clés : Relations Internationales, analyse néogramscienne, Kenneth Waltz, Robert W. Cox

\section{AUTEUR}

\section{FRÉDÉRICK GUILLAUME DUFOUR}

Frédérick Guillaume Dufour termine un doctorat en science politique à l'Université York, Toronto, portant sur la transformation de l'anti-Judaisme médiéval lors de la transition au capitalisme en Europe occidentale. 